\title{
EL ENFOQUe EPISTEMOlÓgICO DE DAVID HilbeRT: EL A PRIORI DEL CONOCIMIENTO Y EL PAPEL DE LA LÓGICA EN LA FUNDAMENTACIÓN DE LA CIENCIA
}

DAVID Hilbert's EPISTEMOlOgicAl APPROACH: THE A PRIORI OF KNOWLEDGE AND THE ROLE OF LOGIC IN FOUNDATION OF SCIENCE

\author{
RODRIGO LOPEZ-ORELLANA \\ Conicyt / Universidad de Salamanca, CHILE / EsPAÑA \\ rodrigo.lopez@usal.es
}

\begin{abstract}
This paper explores the main philosophical approaches of David Hilbert's theory of proof. Specifically, it is focuses on his ideas regarding logic, the concept of proof, the axiomatic, the concept of truth, metamathematics, the a priori knowledge and the general nature of scientific knowledge. The aim is to show and characterize his epistemological approach on the foundation of knowledge, where logic appears as a guarantee of that foundation. Hilbert supposes that the propositional apriorism, proposed by him to support mathematics, sustains - on its turn - a general method for the treatment of the problem in other areas such as natural sciences. This method is axiomatic. Broadly speaking, we intend to recover and update the Hilbert's philosophical thinking about the role of logic for scientific knowledge.
\end{abstract}

Keywords: Hilbert $\bullet$ axiomatic method $\bullet$ concept of proof $\bullet$ foundation of knowledge $\bullet$ logical consistency $\bullet$ logical consequence

\section{Introducción}

El presente trabajo se circunscribe como una investigación en filosofía de la lógica. Dentro de estos límites, exploraremos algunos de los aspectos más relevantes del enfoque de la teoría lógica de la prueba de David Hilbert (1862-1943). Específicamente, nos interesan las consideraciones filosóficas implícitas en su programa. De esta manera, nos centraremos en sus ideas respecto de la lógica, el concepto de prueba, la axiomática, el concepto de verdad, la metamatemática, el conocimiento a priori y la naturaleza general del conocimiento científico; ideas que en los últimos diez años han cobrado gran interés en filosofía de la lógica. Como sabemos, el programa de Hilbert es la base lógico-filosófica del enfoque de teoría de la prueba, especialmente en relación con las nociones de validez, consistencia, proposición y verdad lógica (Corry 2004a, pp.11-20). Esta exploración se vuelve interesante en la medida en que 
rescata la figura de Hilbert como filósofo y en la medida en que actualiza sus reflexiones acerca del papel que juega la lógica para el conocimiento científico, problema aún no resuelto del todo (Haack 2009). Además, contribuye a la escasa literatura en español que hay acerca de su pensamiento.

Con la Conceptografía de Frege nace la idea de que la fundamentación de las proposiciones en toda argumentación (matemática o científica) está sustentada en la fuerza de la prueba lógica, que establece la conexión de verdades a través de las cadenas de inferencia dadas en un argumento. Para Frege, el conocimiento de una verdad científica radica - en última instancia - en una prueba lógica pura, "la cual, prescindiendo de las características particulares de la cosa, solo se funda en las leyes sobre las que descansa todo conocimiento" (Frege 1972, p.3). Así, el análisis de nociones como proposición, verdad, axioma o conocimiento abarca una cuestión filosófica fundamental referente a la relación que se establece entre el lenguaje y el mundo del cual habla. Los diferentes sistemas lógicos formales han intentado capturar dicha relación. En este sentido, propuestas filosóficas como la de Hilbert (también Franz Brentano y Bernard Bolzano) están volviendo a ser estudiadas (Mancosu 2010; Sieg 2013), por ejemplo, para esclarecer aquellas diferencias sustanciales entre lo que se ha llamado enfoques lógicos estáticos y dinámicos (van Benthem 2001; 2014). Además, las ideas de Hilbert siguen siendo muy sugerentes para aquellos enfoques actuales de la filosofía de la ciencia que mantienen un papel central para la axiomatización en la explicación científica y en la caracterización de teorías de las ciencias factuales (da Costa y French 2003; Cassini 2013). ${ }^{1}$

La teoría de la prueba se presenta como un subcampo de la lógica matemática, creado por Hilbert con el objetivo de fundamentar "definitivamente" la consistencia de las matemáticas. Su propósito era completar la formalización de las pruebas matemáticas que había iniciado Frege en 1879 con la formulación de la lógica de primer orden. Su recurso de partida era el tratamiento de las pruebas formales como objetos matemáticos, que son analizables mediante métodos o técnicas que pueden ser empleadas objetivamente por cualquier investigador, sin la necesidad de forzamientos metafísicos ni el uso de conceptos imprecisos. La idea de Hilbert, en sus Fundamentos de la matemática (1927), era abarcar la estructura general de las pruebas matemáticas y de las proposiciones de la lógica. Dicha estructura se construye de acuerdo con las reglas de inferencia y los axiomas de los sistemas, por lo que al enfoque de la teoría de la prueba se le suele llamar también "enfoque axiomático" — axiomatic approach - (Rowe 2000).

La formalización propuesta por Hilbert, a grandes rasgos, establece que las matemáticas deben ser un sistema que parta explícitamente con el listado o vocabulario completo de los símbolos que se van a emplear. Además, debe establecer las reglas de formación para todas las expresiones válidas, para luego especificar las reglas de transformación para pasar de una fórmula válida a otra. Finalmente, seleccionará al- 
gunas expresiones válidas como axiomas, que serán las más importantes para todo el sistema. Todo esto, según Hilbert, entregará una teoría — las matemáticas — de las propiedades combinatorias del lenguaje formal que permitirá hacer afirmaciones verdaderas sobre cualquier expresión determinada del sistema. Sus proposiciones son entonces afirmaciones sobre los símbolos del sistema formal y sobre su disposición o relaciones.

De acuerdo con lo anterior, la prueba de la consistencia de un sistema formal (el principal objetivo) consistiría en demostrar, a partir de enunciados meta-matemáticos finitistas (los axiomas), que nunca puede obtenerse en el sistema una fórmula y su negación (una contradicción). Los desarrollos de la teoría de la prueba en lógica y matemática tienen este punto de partida.

Según algunos autores, como Buss (1998), los objetivos originales de la teoría de la prueba fueron:

a. Formular sistemas lógicos con axiomas apropiados para la formalización de las pruebas matemáticas y para caracterizar lo que pueda resultar de esos axiomas. La idea era poner a prueba a los mismos sistemas formales, es decir, analizar su «resistencia» lógica - "the proof-theoretic strength of formal systems" (Resnik 1974) -

b. Estudiar la estructura formal de las pruebas para encontrar sus formas normales o "regulares" y para establecer los hechos sintácticos acerca de esas pruebas (Hilbert 1950, pp.11-2). ${ }^{2}$ Se entenderán entonces las pruebas como objetos independientes de estudio, como se señaló anteriormente.

En una segunda fase, desde la irrupción de los sistemas computacionales en la segunda mitad del siglo XX, sus objetivos han sido:

c. Estudiar qué tipo de información adicional puede ser extraída de las pruebas formales más allá de la verdad que los teoremas hayan probado. Esta cuestión estriba en que las pruebas pueden contener información computacional o información constructiva, lo que permite que la teoría de la prueba tenga aplicaciones en otros campos gracias a la programación lógica, como es en el caso de la inteligencia artificial. La capacidad de manipulación de las pruebas, su carácter constructivo, posibilita la programación lógica como una herramienta precisa en estos campos. Esto último ha tenido como consecuencia que la teoría de la prueba esté fuertemente ligada a los desarrollos de la computación moderna.

d. Estudiar la mejor forma de construir pruebas formales. En efecto, podemos encontrar aquí todo un campo de desarrollo para lograr máquinas computacionales que puedan generar pruebas cada vez más eficientes. 
Si bien es cierto que fue imposible cumplir el programa de Hilbert, después de las demostraciones de los teoremas de incompletud de $\mathrm{Gödel}^{3}$ que acabaron con todo intento de formalizar completamente la matemática, su influencia para la teoría de la prueba ha sido determinante, marcando su desarrollo posterior y toda su concepción filosófica. Una de las razones que se dan para ello es que, aunque no fue posible formalizar toda la matemática, sí es posible construir sistemas satisfactoriamente formalizados. En efecto, es el caso - por ejemplo - del sistema axiomático ZFC (axiomatización de Zermelo-Fraenkel más el axioma de elección); la teoría axiomática de la verdad Kripke-Feferman; el programa estructuralista en física de Sneed (1971) y sus continuadores; el sistema de lógica proposicional borrosa de Hajek (1998) basic fuzzy propositional logic —; entre muchos. ${ }^{4}$ Las bases asentadas por Hilbert propiciaron esos desarrollos. También se arguye que los sistemas matemáticos sí tienen un conjunto computable de axiomas y es posible hacer pruebas formales de ellos no son triviales - , y se ha demostrado que pueden ser aplicados a otros ámbitos del conocimiento, como la ingeniería informática. La teoría de la prueba - hasta nuestros días - continúa entonces el proyecto de formalización y axiomatización de Hilbert, pero en una versión más "mesurada" (Corry 2004b, pp.172-82).

En lo siguiente, iremos revisando este enfoque lógico teniendo en mente los puntos a. y b., que constituyeron el programa original de Hilbert.

\section{Aclaraciones previas sobre el pensamiento de Hilbert}

Cabe advertir que podríamos reconocer tres periodos o momentos del pensamiento de Hilbert (Detlefsen 1986). Sin embargo, esto es arriesgado y puede conducir a ciertas confusiones en la interpretación de su proyecto general. Siguiendo a algunos autores, por ejemplo Rowe (2000) y Sieg (2013), podemos aventurar lo siguiente.

Un primer periodo de su pensamiento transcurre desde 1888, con su trabajo de demostración del teorema de finitud, pasando por Los fundamentos de la geometría de 1899 hasta su famosa conferencia "Problemas matemáticos" (o también conocida como "Los problemas futuros de la matemática") de 1900. En esta fase presenta su programa formalista, basado principalmente en la exposición de los fundamentos de la matemática a partir del método axiomático formal, con la pretensión de extenderlo para todas las demás ciencias. ${ }^{5}$ Además, en este periodo sustenta — siguiendo a Kant - que los únicos tipos de conocimiento son la experiencia y el pensamiento, siendo este último el único capaz de lograr autonomía y capacidad creativa, superando la fuerza de los datos de los sentidos y los problemas que presenta el conocimiento empírico. Para Hilbert, esta cuestión se refleja en la fuerza del conocimiento matemático puro ${ }^{6}$ y en la capacidad de su método axiomático, que según él es aplicable a toda la ciencia. En este periodo también está lidiando con sus ideas sobre la exis- 
tencia abstracta de los objetos matemáticos, llegando a afirmar que "probar la no contradicción de un concepto prueba también su existencia matemática" - reductio ad absurdum - (Hilbert 2000, pp.414-5). Además, concibe la "prueba" como un objeto lógico, por lo que algunos autores reconocen cierto platonismo en Hilbert (Grattan-Guinness 2000). Estas ideas le llevaron a mantener una interesante polémica con Frege, con Leopold Kronecker ${ }^{7}$ y también con los intuicionistas (cf. Rowe 2000).

Un segundo periodo del pensamiento de Hilbert puede datarse de 1904, con su artículo "Sobre los fundamentos de la lógica y de la aritmética" (Hilbert 1905). Aquí comienza a lidiar con las paradojas que emanan de la teoría de conjuntos y además recibe con gran entusiasmo - pocos años después - los Principia Mathematica de Whitehead y Russell (1910-1913). En esta fase vacila con el rol que debe tener la lógica en la fundamentación matemática. En una primera instancia tiene la idea de que la aritmética tienen un contenido propio, esto es, que la aritmética no puede construirse únicamente con conceptos lógicos, sino necesariamente también a partir de conceptos aritméticos como "número" o "conjunto". En su artículo "Pensamiento axiomático" de 1918 profundiza estas ideas:

De hecho, la aritmética se designa como parte de la lógica y se acostumbra a presuponer, en la fundación de la aritmética, los principios fundamentales tradicionales de la lógica. Pero con una atenta consideración nos daremos cuenta de que en la exposición habitual de las leyes de la lógica ya se están empleando ciertos conceptos fundamentales de la aritmética, por ejemplo, el concepto de agregado [conjunto], y en parte también el concepto de número. Caemos así en un círculo vicioso y, por tanto, para evitar paradojas, será necesario un desarrollo parcialmente simultáneo de las leyes de la lógica y de la aritmética (Hilbert 1905, p.347).

En una segunda instancia, influenciado por los Principia, abandona esta idea de la independencia de la aritmética de la lógica, llegando a sostener que la teoría de los números y la teoría de conjuntos son partes de la lógica misma, lo que se prueba con la axiomatización de la lógica:

Pero como el examen de la consistencia es una tarea que no puede evitarse, parece necesario axiomatizar la lógica misma y probar que la teoría numérica y la teoría de conjuntos son solo partes de la lógica (Hilbert 2005a, p.1113, §39).

Su motivo principal para este giro es la necesidad de mantener la consistencia de las matemáticas en su totalidad. Debido a esto se embarcará en el proyecto de la prueba de su consistencia:

Si la teoría de un campo de conocimiento - es decir, el marco de conceptos que lo representa - debe servir a su propósito de orientar y ordenar, 
entonces debe satisfacer dos requisitos por sobre todo: en primer lugar, debe darnos una visión general de la independencia y de la dependencia de las proposiciones de la teoría; y en segundo lugar, debe darnos una garantía de la consistencia de todas las proposiciones de esa teoría. En particular, los axiomas de cada teoría deben ser examinados desde estos dos puntos de vista (Hilbert 2005a, p.1109, §9).

Un tercer momento del pensamiento de Hilbert puede datarse a partir de su comunicación "Nueva fundamentación de la matemática" de 1922. La diferencia aquí con sus ideas anteriores en "Pensamiento axiomático" es que especificará el papel de la lógica como una importante fuente de conocimiento matemático, pero no la única. Aquí comienza a desarrollar ideas que anteriormente había esbozado en sus diferentes textos pero que no aparecían de manera sistemática. Una de estas ideas es la función de la intuición matemática como fuente abstracta de conocimiento. Esta idea aparecerá aplicada - con cierta confusión al respecto - en sus textos "Fundamentos lógicos de la matemática" de 1923, "Lógica y el conocimiento de la naturaleza" de 1930 y en "Fundamentación de la teoría elemental de los números" de 1931.

Si bien es cierto que podríamos hacer tal distinción de una cierta evolución del pensamiento de Hilbert en tres momentos, no abordaremos el problema siguiendo tal esquema. Una tarea semejante es bastante difícil de sortear debido principalmente a las propias confusiones filosóficas de Hilbert, que se van presentando de "ida y vuelta" en sus propios textos. No obstante, la unidad general de su pensamiento es su concepción con respecto a los fundamentos de la matemática, cuyo objetivo principal es defender nuestra posesión de un conocimiento matemático y de su consistencia.

A continuación, abordaremos los principales planteamientos de su programa general.

\section{El concepto de prueba}

Antes de entrar en las consideraciones filosóficas del enfoque de Hilbert, debemos tener claro que el concepto de "prueba" tiene un papel central en matemáticas y lógica ya que es el medio por el cual se establece la verdad de sus proposiciones. Esto es lo que mantuvo ocupados a los matemáticos desde la filosofía de la matemática de Bolzano de 1837 (Bolzano 1973).

Una prueba consiste en una cadena de símbolos que satisfacen un determinado conjunto de reglas y que demuestran un determinado teorema, que a su vez debe expresarse también como una cadena de símbolos. Decimos que cuando tenemos una prueba hemos asegurado entonces la verdad de una proposición matemática (Hilbert 1993, pp.63-7; Cohen 1989, pp.21-42). 
El concepto de prueba está en el centro del programa de Hilbert. En efecto, afirmaremos entonces aquí que son dos los ejes centrales que articulan su programa: i) el método axiomático, con el cual se formaliza toda la matemática, y ii) la prueba de la consistencia de esa axiomatización. Dicha prueba debe realizarse exclusivamente a partir de métodos "finitarios". Es de esta manera que Hilbert asume el enfoque epistemológico de un "razonamiento finitista", ya que piensa que solo con este tipo de razonamiento nos será posible lograr la justificación de la matemática clásica (Zach 2015) y podremos probar la existencia de los objetos matemáticos. Como ya se mencionó anteriormente, la noción de prueba le sirve a Hilbert para sustentar la existencia de estos objetos: "probar la no contradicción de un concepto prueba también su existencia matemática":

\section{Si se asignan atributos contradictorios a un concepto, digo, que matemática- mente el concepto no existe. Así, por ejemplo, un número real cuyo cuadrado es -1 no existe matemáticamente. Pero si se puede demostrar que los atribu- tos asignados al concepto nunca pueden conducir a una contradicción, por la aplicación de un número finito de procesos lógicos, digo que la existencia matemática de ese concepto (por ejemplo, de un número o una función que satisface ciertas condiciones) queda así demostrada (Hilbert 2000, p.414).}

La idea filosófica general de Hilbert es que para todo tipo de conocimiento debemos ser capaces de introducir nociones abstractas para redondear o asegurar nuestras teorías, pero evitando los peligros inherentes en esa introducción: las paradojas, especialmente para la teoría de conjuntos. Su estrategia es "domar el infinito insistiendo en pruebas de consistencia de los sistemas" (Kitcher 1976, pp.99-100). Abordaremos estas ideas de Hilbert más adelante.

\section{La axiomática}

El proyecto de Hilbert comienza a configurarse desde las Die Grundlagen der Geometrie (1899) - Los fundamentos de la geometría - . Para muchos este es el origen específico del método axiomático moderno. El hilo conductor del proyecto y su método son el mismo que el propuesto anteriormente por Bolzano (1837), Brentano (1874) y Frege (1879): el análisis lógico, ya sea de las proposiciones de la ciencia, del lenguaje o de nuestras percepciones. Este análisis lógico debe ser equivalente al desarrollado en geometría desde Euclides. Para establecer los fundamentos de la matemática - tanto de la geometría como de la aritmética, pero también lo supone para las ciencias naturales (Hilbert 2005c) - debemos partir de un pequeño número de axiomas, principios simples y fundamentales, que permitan reconstruir la totalidad de su estructura consistentemente. Así, en ambas "fundamentaciones" ${ }^{8}$ Hilbert 
comienza por una clasificación de los axiomas sobre los cuales se basa todo el andamiaje. Específicamente, en la Fundamentación de la Geometría se lleva a cabo el análisis lógico de nuestra percepción del espacio gracias a esos axiomas. ${ }^{9}$

Durante el siglo XIX ocurrirá una - casi - "catástrofe" en geometría debido al debilitamiento del edificio de los Elementos de Euclides - paradigma de rigor y exactitud matemática -, principalmente, por los desarrollos de las geometrías no euclídeas a lo largo de ese siglo. No obstante, durante el mismo período se renueva el interés por la axiomática y comienzan a prepararse proyectos de fundamentación de la geometría. El primer paso contundente y auspicioso lo da Giuseppe Peano (1858-1932) desde la aritmética, con su artículo de 1889 "Nuevo método de exposición de los principios de la aritmética", gracias a la entrega de un sistema completamente abstracto sustentado en axiomas específicos en un lenguaje lógico - simbólico - , del cual Frege y Hilbert afirmarán sus propias propuestas. La idea general es lograr sustentar una estructura matemática consistente, poniendo énfasis en los axiomas como principios simples, pero fundamentales y seguros, para construir el pretendido edificio sólido de las matemáticas. Por este motivo, a la visión de las matemáticas que tenía Hilbert se le ha calificado también como "estructural" (Corry 2004b, pp.137-82).

El sistema de axiomas simple y completo de Hilbert pretende probar todos los teoremas tanto de la geometría como de la aritmética realizando un giro conceptual muy importante: deja en "suspensión teórica" las definiciones del sistema. Por ejemplo, en el caso de la geometría de Euclides aparta las 23 definiciones "intuitivas" (v.g., plano, recta, punto), que dejarán de tener una relevancia matemática para la configuración del sistema. Recordemos que el primer libro de los Elementos se compone de:

- 23 definiciones (v.g., punto, línea, plano).

- 5 postulados (v.g., "por dos puntos diferentes pasa una sola línea recta").

- 5 nociones comunes (v.g., "cosas iguales a una tercera son iguales entre sí").

- 48 proposiciones (v.g., "si dos triángulos tienen dos lados respectivos iguales, y tienen los ángulos comprendidos iguales, también tendrán las bases iguales, y los triángulos serán iguales, y los ángulos restantes serán iguales, concretamente los opuestos a los lados iguales").

Euclides llega a demostrar en total unas 465 proposiciones verdaderas en los Elementos, a partir de leyes lógicas básicas (como el principio de identidad) que están implícitas, aunque algunas las expresa en la forma de nociones comunes. Pero bajo la deducción lógica, que nos brinda "supuestamente" un lugar confortable de seguridad y exactitud, puede aparecer un problema difícil de sortear. Debemos recordar también que las definiciones de Euclides son siempre frases breves y precisas que introducen conceptos sin explicaciones ni ejemplos. En otras palabras, sirven para 
introducir los objetos matemáticos al sistema, pero lamentablemente esto no presupone su existencia. Una definición que se suele citar como ejemplo del problema es la definición número 20 del Libro I, donde se introducen los triángulos equiláteros (Mueller 1981, pp.11-25):

Def. 20. De entre las figuras triláteras, triángulo equilátero es la que tiene los tres lados iguales, isósceles la que tiene solo dos lados iguales, y escaleno la que tiene los tres lados desiguales (Euclides 1991, p.195).

Pero la cuestión es que estos son objetos que Euclides solo utiliza después de haber demostrado que se pueden construir en la primera proposición:

Prop. 1. Construir un triángulo equilátero sobre una recta finita dada (Euclides 1991, p.201).

Para llevar a cabo la prueba deberá partir de la definición de triángulo:

Def. 19. Figuras rectilíneas son comprendidas por rectas, triláteras las comprendidas por tres, cuadriláteras las comprendidas por cuatro, multilateral las comprendidas por más de cuatro rectas (Euclides 1991, p.195),

junto con la noción de "segmentos iguales" para obtener los conceptos de triángulo equilátero, isósceles y escaleno. Y aun así esta introducción es sencilla. Más difícil es introducir:

Def. 1. Un punto es lo que no tiene partes [...]

Def. 4. Una línea recta es aquella que yace por igual respecto de los puntos que están en ella (Euclides 1991, pp.189-90).

Podríamos preguntarnos, por ejemplo, ¿qué quiere decir que no tenga partes? ¿Hay mejores definiciones que esa: v.g., "el corte de dos líneas"? ¿Qué significa "yacer por igual"? ¿Qué sustenta la comprensión y la existencia de esos objetos abstractos? Las dificultades ontológicas y epistemológicas son evidentes para un análisis más fino. Para Hilbert, la fundamentación de la geometría (y de las matemáticas en general) requiere de una configuración de sistema robusto sin grietas. Su propuesta axiomática intenta seguir adelante apartando nociones o conceptos problemáticos que impidan alcanzar la consistencia interna de los sistemas matemáticos. De ahora en adelante, importarán únicamente las relaciones que se establezcan entre los axiomas. Los axiomas pasarán a ser ahora las nociones implícitas que pueden sustentar dichas definiciones.

Hilbert nos pone el siguiente ejemplo. Consideremos tres distintos sistemas de cosas. El primer sistema se compone de cosas que llamaremos 'puntos', que designaremos con las letras $A, B, C, \ldots$ El segundo sistema contiene las cosas que llamaremos 
líneas rectas, que designaremos con las letras $a, b, c, \ldots$ Finalmente, el tercer sistema se compone de las cosas que llamaremos planos, designadas con las letras griega $\alpha, \beta, \gamma, \ldots$ Los puntos son los elementos de la geometría lineal; los puntos y líneas rectas son los elementos de la geometría plana; y los puntos, líneas y planos son los elementos de la geometría del espacio o los elementos del espacio. Pensamos que entre estos puntos, líneas rectas y planos existen ciertas relaciones mutuas, que indicamos a través de palabras como 'se encuentra', 'entre', 'paralelo', 'congruentes', 'continua', entre otras. La descripción completa y exacta de todas esas relaciones se deduce como consecuencia de los axiomas de la geometría (Hilbert 1950, p.2).

Es necesario recordar que para Euclides los axiomas son esos postulados y nociones comunes que se entienden como dos series de propiedades de los objetos matemáticos aceptados sin discusión, por intuición. Son afirmaciones generales válidas, para todo el conocimiento científico, cuya evidencia las hace intuitivamente aceptables generalmente - y no necesitan una demostración, como las nociones comunes No. 1. "las cosas iguales a una misma cosa son también iguales entre sí" y No. 7. "las cosas que coinciden entre sí son iguales entre sí" (Euclides 1991, pp.199-201). Las proposiciones, o teoremas, son los enunciados que se logran probar gracias a los principios o axiomas aceptados y a las propiedades de los objetos abstractos supuestas en las definiciones; partiendo también de las proposiciones anteriores probadas como verdaderas.

No obstante, para Hilbert, solo el sistema de axiomas puede determinar todos los objetos matemáticos considerados gracias a la fuerza de las pruebas en el mismo sistema. Esto quiere decir que lo que sustenta a dichos axiomas no es la intuición, como en Euclides, sino que son las consecuencias que se siguen dentro del sistema por las relaciones que este mismo establece entre sus axiomas. Así, en ambas "fundamentaciones", comienza por analizar todas las consecuencias que se siguen de las relaciones de esos axiomas, hasta llegar a la cuestión más importante que es el estudio de los problemas de independencia de los axiomas y su consistencia, esto es, para todas las proposiciones matemáticas. Según Hilbert, este será el único camino para una fundamentación de las matemáticas. Según Mueller (1981, pp.11-25), Hilbert, influenciado por su trabajo sobre las bases del cálculo matemático - con "cálculo" se refiere aquí a la aritmética -, vio la axiomatización rigurosa como una característica necesaria de las matemáticas. Esto lo llevó a eliminar de las matemáticas la necesidad de confiar en la simple intuición para las pruebas.

Para Hilbert, tal reformulación de los axiomas es la única formulación lógica posible. La estructura lógica de las matemáticas y su funcionamiento mostrarían perfectamente la consistencia del sistema, sin necesidad de saber lo que significan los axiomas, o los conceptos o nociones que comportan. No es necesario acudir a la intuición para obtener su evidencia o sustentar la demostración de las proposiciones a las que llega. El puro mecanismo o funcionamiento de los axiomas y de las reglas 
lógicas revela esta cuestión. El razonamiento debería ser llevado a cabo únicamente de acuerdo con las normas puramente mecánicas de la prueba, sustentándose en la verdad de los axiomas. En geometría nos basta solo con aplicar las reglas a los axiomas, de una manera ciega o confiada, sin necesidad de saber lo que significan, y de esta manera obtener todo el conocimiento geométrico. Claro está que no sin entender nada en absoluto, sino más bien confiados en la secuencia lógica de las proposiciones — sin necesidad de recurrir a ninguna intuición — que descansa a su vez en la verdad de los axiomas: "Uno podría darle los axiomas a una máquina lógica, por ejemplo al piano lógico ${ }^{10}$ de Williams Stanley Jevons, y vería salir de ella toda la geometría" (Mueller 1981, p.6).

Veamos a continuación, brevemente, cómo Hilbert prueba estas cuestiones. Por ejemplo, para el caso de la aritmética, en los Fundamentos de la matemática, los axiomas son presentados con la siguiente clasificación clásica de la geometría (Hilbert 1993, pp.18-20):

1. Axiomas de conexión

1.1 A partir de dos números $a$ y $b$ podemos obtener por "adición" otro número $c$. Simbólicamente $a+b=c$ o bien $c=a+b$

1.2 Dados dos números $a$ y $b$ cualesquiera, existe un único número $x$ y existe un único número $y$ tales que $a+x=b$ y $y+a=b$

1.3 Existe un número definido 0 con la propiedad de que, para todo número $a$

$a+0=a$ y $0+a=a$

1.4 Dados dos números $a$ y $b$ cualesquiera, podemos obtener por "multiplicación" otro número $c$. En símbolos $a \cdot b=c$ o bien $c=a \cdot b$

1.5 Si $a$ y $b$ son números y $a$ es distinto a 0 , existe un único número $x$ y un único número $y$ tales que $a \cdot x=b$ y $y \cdot a=b$

1.6 Existe un número definido 1 con la propiedad de que, para todo número $a$,

$a \cdot 1=a$ y $1 \cdot a=a$

2. Axiomas para las operaciones

$2.1 a+(b+c)=(a+b)+c$

$2.2 a+b=b+a$

$2.3 a \cdot(b \cdot c)=(a \cdot b) \cdot c$ 
$2.4 a \cdot(b+c)=a \cdot b+a \cdot c$

$2.5(a+b) \cdot c=a \cdot c+b \cdot c$

$2.6 a \cdot b=b \cdot a$

3. Axiomas de orden

3.1 Si $a$ y $b$ son dos números distintos cualesquiera, uno de ellos (por ejemplo, $a$ ) es mayor ( $>$ ) que el otro; este último es menor que el primero. En símbolos $a>b$ y $b<a$

3.2 Si $a>b$ y $b>c$, entonces $a>c$

3.3 Si $a>b$, entonces $a+c>b+c$ y $c+a>c+b$

3.4 Si $a>b$ y $c>0$, entonces $a \cdot c>c \cdot b$ y $c \cdot a>c \cdot b$

4. Axiomas de continuidad

4.1 (Axioma de Arquímedes) Sean $a$ y $b$ dos números cualesquiera, $a>0 \mathrm{y}$ $b>0$. Sumando $a$ consecutivamente, puede obtenerse una suma con la propiedad tal que

$a+a+\cdots+a>b$

4.2 (Axioma de completud) Si al sistema de los números se añade otro sistema de objetos, entonces en el nuevo sistema no pueden ser válidos los axiomas 1., 2., 3. y 4.1. En otras palabras, los números conforman un sistema de objetos que tiene la propiedad de hacer imposible una extensión [propia] del mismo conservando la validez de la totalidad de sus axiomas.

Hilbert nos dirá entonces que algunos axiomas pueden obtenerse a partir de los demás: 1. 1-6, 2. 1-6, 3. 1-4 y 4. 1-2. Esto plantea el problema de su independencia lógica. Y además se pueden también obtener algunos hechos matemáticos relevantes, como los siguientes:

a. La existencia del número 0 (axioma 1.3) es una consecuencia lógica de los axiomas $1.1,1.2$ y 2.1 , que será un principio que dependerá esencialmente de la ley asociativa para la adición.

b. La existencia del número 1 (axioma 1.6) se obtiene a partir de los axiomas $1.4,1.5$ y 2.3 , principio relacionado esencialmente con la ley asociativa para la multiplicación.

c. La ley conmutativa para la adición es una consecuencia de la ley asociativa para la adición y las leyes distributivas: resulta de los axiomas 1, 2.1, 2.4 y 2.5.

d. La ley conmutativa para la multiplicación (axioma 2.6) se obtiene de los axiomas 1, 2. 1-5, 3. y 4.1, pero no se puede deducir solo de los axiomas 1., 2. 1-5 y 
3. Podremos llegar a este principio si se añade también el axioma 4.1 (axioma de Arquímedes).

e. Los axiomas 4.1 y 4.2 son independientes entre sí y nada nos informan acerca del concepto de convergencia ni sobre la existencia de un límite.

Estas relaciones e independencias de ciertos axiomas se pueden probar de la siguiente manera. Para el caso de la ley conmutativa de la adición, Hilbert realiza la siguiente prueba.

Prueba:

Tenemos que

$$
\begin{aligned}
(a+b) \cdot(1+1) & =(a+b) \cdot 1+(a+b) \cdot 1=a+b+a+b \\
& =a \cdot(1+1)+b \cdot(1+1)=a+a+b+b,
\end{aligned}
$$

por lo que

$$
a+b+a+b=a+a+b+b
$$

y, por lo tanto, según 1.2 ,

$$
b+a=a+b
$$

Según Hilbert, las relaciones que se plantean con este sistema, específicamente sobre los números reales, son todas finitas. En este caso, toda relación de existencia de conjuntos infinitos de números queda descartada. Hilbert entenderá por conjunto de números reales no la totalidad de las leyes posibles según las cuales pueden avanzar los elementos de una sucesión fundamental, sino más bien un sistema de objetos cuyas relaciones se encuentran determinadas por el sistema cerrado y finito de todos los axiomas antes descritos. En relación con este sistema cerrado y finito, ninguna afirmación será válida si no puede deducirse a partir de esos axiomas por medio de un número finito de inferencias lógicas (Hilbert 1993, pp.21-2).

Para probar la independencia y consistencia de su sistema, Hilbert parte de la construcción de modelos. Probar la independencia del axioma $X$ respecto del sistema de axiomas $S$ significa que el sistema $T$ obtenido añadiendo a $S$ la negación del axioma $X$ es consistente. Para lograr esto, se construye un modelo que verifica el sistema $S$ de axiomas y la negación del axioma $X$. Así, la existencia de una contradicción en $T$ implicaría una contradicción en las proposiciones obtenidas dentro del modelo construido, y por tanto en la teoría con la que se ha construido el modelo (Corry 2004a, pp.9-99).

Una de las conclusiones más relevantes de este método, y que resulta importante para nuestro trabajo, es que las proposiciones de las matemáticas (como las de la geometría euclidiana) se prueban de manera tal que la misma prueba muestra precisamente que los axiomas subyacen y hacen posible la demostración. Así, según 
Hilbert, las matemáticas dan una solución precisa a todo problema a través de una prueba, que muestra la posibilidad o imposibilidad de su solución gracias a los axiomas. Hilbert sostendrá entonces que la consistencia de un sistema es la prueba de la existencia misma de los objetos que trata. Los axiomas que subyacen a la estructura lógica finita de la matemática, o del sistema, son verdaderos en tanto que son parte de esa misma estructura consistente. ${ }^{11}$

Remarcando esta concepción de Hilbert de las matemáticas, las cuestiones centrales que aborda la teoría de la prueba son la independencia (de los axiomas) y la consistencia (del sistema): esto es la axiomática. Esto es lo que caracteriza a su proyecto. En otras palabras, el método conlleva en sí la exigencia de que se demuestre la independencia y completud de los axiomas, y en especial que pruebe la no contradicción. Probar la no contradicción de un concepto es probar a su vez la existencia matemática de este. El formalismo que se le atribuye a Hilbert debe estar ligado a esta idea: la necesidad de definir y comprender mejor las teorías matemáticas y científicas existentes a partir de sistemas axiomáticos abstractos y de sus análisis que se sustentan en una estructura elaborada y establecida de entidades matemáticas. Según Hilbert, el desarrollo de la ciencia involucra entonces «una extensión en alcance y una clarificación en curso de la estructura lógica de sus piezas existentes» (Corry 2004b, pp.161-2).

Una cuestión interesante con respecto al concepto de consistencia es que Frege no estuvo para nada de acuerdo con Hilbert en este punto. Como sabemos, ambos mantuvieron una rica comunicación por carta a partir de 1899, a raíz de las preguntas y críticas que Frege le hace sobre su lectura de la Fundamentación de la geometría (Hintikka 1988). Frege consideró inútil ofrecer una prueba de consistencia de los axiomas, ya que para él "la consistencia se sigue de la verdad y los axiomas son por definición verdaderos" (Gabriel 1980, pp.35-8. Carta de Frege a Hilbert del 27 de diciembre de 1899). Para Frege los axiomas son proposiciones verdaderas pero indemostradas. Pensaba que de la verdad de los axiomas ya se sigue que estos no se contradicen entre sí, por ello era infructuoso y superfluo la demostración de su consistencia. A lo que Hilbert contestó:

He estado diciendo exactamente lo contrario: si los axiomas arbitrariamente dados no se contradicen entre sí con todas sus consecuencias, entonces son verdaderos y las cosas definidas por los axiomas existen. Este es para mí el criterio de la verdad y la existencia (Gabriel 1980, pp.39-40. Carta de Hilbert a Frege del 29 de diciembre de 1899).

Según Mosterín (1980, p.298), el problema de Hilbert fue equiparar consistencia y verdad: si los axiomas no son contradictorios, son consistentes, pero no por ello verdaderos. Los axiomas de Hilbert, en sentido estricto, no son entidades de las cuales cabe predicarse la verdad o la falsedad. 
Frege tampoco aceptó la noción de prueba de Hilbert. Para él las pruebas abstractas, tal como las describe Hilbert, no deben aceptarse. En primer lugar, porque parten de axiomas abstractos. Estos resultan ser simplemente proposiciones que no expresan idea alguna - que en términos de Frege diríamos "pensamiento" —, y una inferencia solo puede partir de una idea para llevar a otra. Frege llega a decir que tal tipo de axiomas no pueden ser premisas de una cadena de inferencias. En efecto, no era partidario de aceptar pruebas que se obtengan exclusivamente a partir de las reglas de inferencias dadas en un sistema, ya que afirmaba la necesidad del análisis de la verdad en el juicio. La verdad en el juicio está garantiza por el contenido proposicional del mismo que es su valor de verdad. Por ello, toda inferencia parte de una verdad reconocida previamente en la cadena argumentativa intersubjetiva, que describe en su ontología de objeto y función - que aquí solo mencionaremos (Blanchette 2012). En definitiva, para Frege la prueba de la consistencia no es garantía de existencia de los objetos abstractos lógico-matemáticos. Para Frege, el recurso a construcción de modelos - que explicamos anteriormente - solo traería confusiones; tales objetos abstractos no garantizan la obtención de la estructura finita de las matemáticas. Por ello desarrollará, posteriormente, un análisis de la verdad a partir de la noción de sentido y referencia.

\section{La verdad y la base lógica de las matemáticas}

Para redundar en estas ideas y para una solución a los problemas planteados por Frege, Hilbert aplicará el método axiomático para las fundamentaciones de la geometría, la aritmética, la mecánica y la probabilística. La axiomática será para él aquello que confiere un mayor grado de certidumbre al conocimiento humano. Entendió así que los principios axiomáticos proveen conceptos matemáticos justificados y, aún más, con existencia independiente. Podemos decir entonces que Hilbert elaboró una concepción de la verdad matemática equiparada con la de consistencia lógica. Según Hilbert (2000, p.414), investigar los fundamentos de una ciencia significa establecer un sistema de axiomas que contiene una descripción exacta y completa de las relaciones que subsisten entre las ideas elementales de esa ciencia. Así mismo, los axiomas serán las definiciones de esas ideas elementales, y ningún enunciado dentro del campo de esa ciencia - cuya fundamentación estamos probando - se sostiene como correcto a menos que pueda derivarse de esos axiomas por medio de un número finito de pasos lógicos.

A partir de - lo que hemos identificado como - su "segundo periodo" de pensamiento, específicamente con sus trabajos "Pensamiento axiomático" (1918), "Nueva fundamentación de las matemáticas" (1922) y "La fundamentación lógica de las matemáticas" (1923), Hilbert afirmará que la verdad de la matemática está sustentada 
entonces en la verdad lógica. La razón es que, al reconocer el método axiomático como el único camino para la fundamentación de las matemáticas - y para el conocimiento científico en general - , está obligado a reconocer que la axiomatización de la lógica prueba que la teoría de los números y de los conjuntos son una parte de la lógica. Negará entonces que la aritmética tenga un contenido a priori propio. Supone, al igual como ya lo habían adelantado Bolzano, Boole y Peirce ${ }^{12}$ que la lógica parece ser la única fuente segura de las matemáticas, es el único saber que garantiza un conocimiento abstracto y consistente. Incluso ya en "Sobre el concepto de número" (de 1900) establecía esa relación estrecha entre axiomática y lógica: "el método axiomático tiene la ventaja de proporcionar una exposición concluyente y la plena confianza lógica al contenido de nuestros pensamientos" (Hilbert 1900, citado por Corry 2004b, p.163).

En general, los axiomas pueden describir cada una de las relaciones que existen entre todos los conceptos primitivos de la ciencia, y luego así definirlos explícitamente. Las relaciones descritas son relaciones lógicas y las proposiciones verdaderas de una ciencia en particular son consecuencia lógica de los axiomas escogidos. Por ejemplo, respecto del campo de la física dirá que las contradicciones que surgen en las teorías físicas siempre pueden ser eliminadas al cambiar los axiomas; la dificultad estriba en hacer que todas las leyes físicas observadas sean ahora consecuencias lógicas de esos nuevos axiomas que se han elegido (Hilbert 2005a, p.112).

Respecto de los fundamentos de la matemática, Hilbert intentará eliminar, de una vez por todas, la duda general sobre la fiabilidad de las inferencias matemáticas. El primer paso, como ya se señaló anteriormente (en el caso de la geometría), es dejar las intuiciones. Hilbert sostenía que las dificultades de las matemáticas podían ser resueltas con una teoría de la prueba como fundamento inobjetable, principalmente para la teoría de conjuntos. La estrategia de Hilbert es comenzar con el problema más clásico de esta teoría que es el problema del continuo. El enfrentamiento con este problema podría vislumbrar las soluciones a una teoría de la fundamentación en general.

\section{Hilbert y el problema del continuo}

La estrategia es llegar al problema a partir de la formulación del axioma de elección de Zermelo, enunciado en 1904, quien había entregado por primera vez una prueba de la buena ordenación del continuo. Su propósito era dar respuesta a la pregunta de Georg Cantor (1845-1918) - hipótesis del continuo - sobre la existencia de algún conjunto infinito cuyo cardinal sea estrictamente mayor que el cardinal de los números naturales $\aleph_{0}$ y a la vez estrictamente menor que el cardinal mayor de los números reales con infinitos decimales $\aleph_{1}$ (Huertas y Manzano 2017, pp.4-8). 
Hilbert nos dice en su conferencia Problemas matemáticos (1900) que este seguía siendo uno de los problemas matemáticos más importante y abiertos (sin solución) para las matemáticas modernas. Era el primer problema de la lista que presenta en dicha conferencia. Para Hilbert, los más sugerentes y notables logros del siglo XIX en este campo son la formulación aritmética del concepto del continuo en las obras de Cauchy, Bolzano y Cantor, y el descubrimiento de la geometría no euclidiana por Gauss, Bolyai y Lobachevsky (Hilbert 2000, pp.412-3).

En La fundamentación lógica de las matemáticas (1923), para presentar su teoría de la prueba, Hilbert comienza señalando que el análisis lógico de la especie estudiada en su teoría mostraba que el pensamiento esencial que subyace al principio de elección es un principio lógico general, que es necesario e indispensable, incluso para los rudimentos más elementales de la inferencia matemática. Creyó firmemente que su teoría hacía seguros tales rudimentos y al mismo tiempo que establecía el principio de elección (Hilbert 2005c, 1137).

Recordando el problema que trae a colación Hilbert - muy brevemente - en la teoría de conjuntos axiomática el axioma de elección está incluido en la formalización de esta teoría. El axioma postula que, para cada familia de conjuntos no vacíos, existe otro conjunto que contiene un elemento de cada uno de aquellos. Su formalización tenía como objetivo solucionar los problemas con el concepto de infinito actual ligado al concepto de conjunto y a los problemas de buen orden. Zermelo propuso que un "conjunto bien ordenado" es un conjunto no vacío totalmente ordenado tal que todo subconjunto no vacío tiene un elemento mínimo. Podemos decir que un conjunto $A$ es bien ordenado si y solo si es totalmente ordenado y bien fundado. Zermelo lo formuló entonces como principio, principio del buen orden, que establece que todo conjunto que esté formado únicamente por números naturales tiene un primer elemento. Es decir, que el conjunto de los números naturales es bien ordenado. El primer elemento de los números naturales es 0 (Huertas y Manzano 2017, pp.23-36).

Antes de la axiomática de Zermelo y Fraenkel, Cantor había creído proporcionar uno de los métodos más efectivos para tratar el problema a partir de la axiomatización de los sistemas y concibiendo un conjunto como una sola entidad matemática que a su vez puede ser contenida por otro conjunto. El problema con este concepto era su imprecisión en la definición, aunque la idea intuitiva de "universo" o "dominio" de objetos, a partir del cual se construyen los conjuntos, tuvo siempre una plena aceptación entre los matemáticos (Grattan-Guinness 2000). De la misma manera era aceptado tratar esa entidad como un objeto singular. Cantor apelará al estudio únicamente de las relaciones que se establecen entre estos objetos matemáticos, sin importar de qué naturaleza sean. El comportamiento que tiene un conjunto establece su aceptación como entidad matemática, lo que se prueba con las relaciones de pertenencia de los elementos $(\in)$. Zermelo sigue estas ideas de Cantor, pero sustenta la solución en la axiomatización de la teoría para probar la existencia de estos objetos 
y de sus relaciones (especialmente de las funciones de elección y buen orden). Zermelo creía que la única forma de evitar las paradojas, como la paradoja de Russell (1902) (Gabriel 1980) - a la que se vio enfrentado sin éxito Frege - era probando la consistencia del axioma de elección ya que así todo lo que podamos probar de la teoría de conjuntos sería verdadero. Todo esto, obviamente, antes del advenimiento del teorema de Gödel (Grattan-Guinness 2000, pp.507-13).

La formulación del axioma de elección es la siguiente:

$$
\begin{array}{r}
\forall A(\emptyset \in A \wedge \forall x y(x \in A \wedge y \in A \wedge x \neq y \rightarrow x \cap y=\emptyset) \rightarrow \\
\exists B \forall z(z \in A \rightarrow \exists ! v(v \in z \cap B)))
\end{array}
$$

Este axioma asegura - en la teoría de Zermelo-Fraenkel — la existencia de un conjunto $B$ obtenido a partir de una colección cualquiera $A$ de conjuntos no vacíos y disjuntos dos a dos. De cada conjunto de $A$ elige un único conjunto para poner en $B$.

Otra formulación que se ha dado es: dado un conjunto $A$ de conjuntos no vacíos y disjuntos, hay un conjunto que tiene un elemento de cada conjunto de $A$. Para esta idea Russell tenía el ejemplo de los calcetines: "dada una colección infinita de pares de calcetines, el axioma de elección nos permite elegir uno de cada par" (Huertas y Manzano 2017, p.36).

Hilbert recurre al teorema de elección de Zermelo para presentar un punto importante en su teoría de la prueba: mostrar que el pensamiento esencial que subyace a un principio matemático es un principio lógico general, necesario e indispensable, para todas las inferencias de la matemática. Afirma que su teoría establece este principio lógico y también todos los principios matemáticos - que son consecuencia de los primeros - . La idea central de su teoría es que todo lo que previamente se ha realizado en las matemáticas deberá ser rigurosamente formalizado. Esto quiere decir que, a partir de entonces, las matemáticas deberán entenderse ahora como un "depósito de fórmulas", donde habitan primeramente las fórmulas con signos lógicos para "implica" $(\rightarrow)$ y "no" $(\sim)$ y luego las fórmulas matemáticas ordinarias. Las fórmulas que sirven como ladrillos de construcción para el edificio son los axiomas. Una prueba tendrá esta figura:

$$
\begin{gathered}
\phi \\
\phi \rightarrow \psi \\
\hline \therefore \psi
\end{gathered}
$$

donde, en cada caso, cada una de las premisas será o un axioma, o un resultado directo de un axioma por sustitución, o estará de acuerdo con la fórmula final $\Lambda$ de una inferencia que debe aparecer anteriormente en una prueba, o que resulte de una fórmula por sustitución. Una fórmula será llamada 'probable' (o ‘demostrable') 
si bien es un axioma, o bien deriva de un axioma por sustitución, o bien es la fórmula final de una prueba (Hilbert 2005d, p.1137).

Según Hilbert, si cumplimos con este requisito y llevamos a cabo la formalización de las matemáticas bajo esta forma conceptual de la prueba $(\phi \wedge(\phi \rightarrow \psi)) \rightarrow \psi$, descrita anteriormente, tendremos una nueva matemática. La formalización de las matemáticas la entenderá como meta-matemática, necesaria para asegurar las matemáticas. La metamatemática que obtenemos tiene por objetivo aplicar la forma conceptual para probar la consistencia de todos los axiomas. El estudio de las matemáticas es entonces el estudio de las pruebas matemáticas "adecuadas", formalmente adecuadas, que son pruebas en sí mismas. Estos son los objetos lógico-matemáticos que sustentan el sistema.

A partir de esto último, según Hilbert, el desarrollo de la ciencia matemática como un todo se lleva a cabo de dos maneras, que se alternan constantemente: por un lado, se derivan nuevas fórmulas de los axiomas a partir de la inferencia formal, por el otro, adjuntamos nuevos axiomas y probamos su consistencia por medio de la inferencia de contenido (Hilbert 2005d, p.1138).

Todos los axiomas y teoremas matemáticos demostrables, que tienen la interacción descrita en el párrafo anterior, son las imágenes de los pensamientos que componen el procedimiento tradicional de las matemáticas. Pero en sí mismas no son verdades absolutas. Las verdades absolutas únicamente son las que aporta la teoría de la prueba a la consistencia de estos sistemas formales. Hilbert señala que los axiomas del programa no pueden ser sino estos:

1. Axioma de implicación

$$
\begin{array}{ll}
A \rightarrow(B \rightarrow A) & \text { Adjunción de una presuposición } \\
\{A \rightarrow(A \rightarrow B)\} \rightarrow(A \rightarrow B) & \text { Cancelación de una presuposición } \\
\{A \rightarrow(B \rightarrow C)\} \rightarrow\{B \rightarrow(A \rightarrow C)\} & \text { Intercambio de presuposición } \\
(B \rightarrow C) \rightarrow\{(A \rightarrow B) \rightarrow(A \rightarrow C)\} & \text { Eliminación de un enunciado }
\end{array}
$$

2. Axioma de negación

$$
\begin{array}{ll}
A \rightarrow(\sim A \rightarrow B) & \text { Ley de contradicción } \\
(A \rightarrow B) \rightarrow\{(\sim A \rightarrow B) \rightarrow B\} & \text { Principio de tertium non datur }
\end{array}
$$

3. Axioma de igualdad

$$
\begin{aligned}
& a=a \\
& a=b \rightarrow(A(a) \rightarrow A(b))
\end{aligned}
$$

4. Axioma de número

$$
\begin{aligned}
& a+1 \neq 0 \\
& \delta(a+1)=a
\end{aligned}
$$

Para Hilbert, la teoría elemental de los números se puede obtener a partir de estos 
axiomas, por medio de su lógica finita y del pensamiento intuitivo puro. Además, se puede probar la existencia de los números enteros positivos y todas sus ecuaciones numéricas. La inferencia lógica sustenta esta obtención. Todas las fórmulas demostrables que se obtienen comportan un carácter finito, esto quiere decir que se obtienen a partir de este número finito de axiomas. Proyecto que ya habían prefigurado Frege y Peano. Pero Hilbert intentará también ir más allá de este dominio de una teoría de lógica finita. Se sumaba a uno de los proyectos más ambiciosos en matemáticas, desde la antigüedad con Zenón de Elea, que era enfrentarse con las dificultades del concepto del infinito. Su idea era lograr superar la intuición concreta o natural y esa noción de finitud lógica, con el objetivo de obtener así fórmulas demostrables que puedan ser "imágenes" de los teoremas transfinitos de las matemáticas ordinarias.

Como señalamos anteriormente, Cantor había introducido - en dos artículos en los Mathematische Annalen en 1895 y 1897, ambos bajo el título "Beiträge zur Begründung der transfiniten Mengenlehre"13 — una teoría de estos objetos matemáticos llamados números "transfinitos" para referirse a los ordinales infinitos, aquellos que son mayores que cualquier número natural. Su idea era poder analizar el problema de la naturaleza de los números irracionales que la matemática no había podido resolver por las confusiones con el concepto del infinito. La solución fue desarrollar una teoría de los números transfinitos a partir del concepto de conjuntos como una colección de objetos concretos o abstractos definibles y distinguibles, considerada como un todo (Cantor 1955, pp.4-82). Hilbert también intentó con su sistema de axiomas entregar una formalización a la teoría de los números transfinitos, para poder sentar sobre ella una mejor base para la teoría de conjuntos, cuestión que como ya habíamos anunciado anteriormente - gracias la paradoja descubierta por Russell en 1901, se había convertido en el proyecto tanto de Hilbert como de Russell y de Zermelo.

La solución de Hilbert era aplicar su teoría fundamental de los números por medio de una lógica finita también para los números transfinitos, sin necesidad de aplicar ningún modo dudoso o problemático de inferencia. Para Hilbert, la verdadera demostración del poder de su teoría de la prueba vendrá cuando, después de haber añadido ciertos axiomas transfinitos, seamos capaces de dar la prueba total de su consistencia. No obstante, Hilbert se pregunta cómo podemos comenzar a separarnos de la intuición concreta y de ese finito para lograr asir el dominio transfinito de la realidad. El punto de partida debía ser la aplicación de los cuantificadores lógicos. Resumiendo su idea, la cuestión es comprender que la afirmación de la existencia de una totalidad finita contable de los objetos que poseen una propiedad particular es lógicamente equivalente a la conjunción de varias afirmaciones individuales. Por ejemplo, 'todas las sillas en este auditorio son de madera' equivale a 'esta silla es de madera, aquella silla es de madera y ... la última silla que está allá es de madera'. Del mismo modo, la afirmación de que existe un objeto con una propiedad en una 
totalidad finita de objetos es equivalente a una disyunción de las afirmaciones individuales, por ejemplo: 'existe entre todos estos pedazos de tiza uno que es de color rojo', equivale a decir 'este pedazo de tiza es rojo o ese pedazo de tiza es rojo...o aquel pedazo de tiza es rojo'. La misma idea podía llevarse a la comprensión de los transfinitos, tratándolos como conjuntos de elementos existentes abstractos. Hilbert aplicará este análisis para la prueba del axioma de elección de Zermelo. El artículo completo "La fundamentación lógica de las matemáticas" de 1923 es una demostración de su método.

\section{La importancia de las matemáticas y de la lógica para la totalidad del conocimiento científico}

De acuerdo con lo que hemos esbozado hasta ahora, ya estamos en condiciones de resumir la concepción epistemológica de Hilbert. Podemos encontrar sus planteamientos, escritos de manera muy explícita, en su trabajo Lógica y el conocimiento de la naturaleza de 1930. En efecto, en este trabajo Hilbert muestra su amplio conocimiento filosófico. Trata aquí el viejo problema filosófico sobre cómo el pensamiento y la experiencia participan juntos en el conocimiento. Hilbert cree que estamos capacitados para responder mejor a ese problema ya que ahora tenemos a favor todos los resultados de la experimentación y las teorías que nos ha proporcionado la ciencia hasta ahora. Pero, además, reconoce que estamos en posición de una ciencia fundamental que ha hecho avances determinantes para el conocimiento en general, la lógica. Esta ciencia nos puede entregar un método general para el tratamiento de ese viejo problema filosófico sobre el conocimiento y, además, puede presentarnos soluciones precisas al tratamiento teórico de los problemas de las ciencias naturales. Hilbert estaba convencido de que el método axiomático pertenecía a la lógica. Para él, la lógica de su época era una ciencia que ya había avanzado lo suficiente y se demostraba en el mismo hecho de habernos dotado de un método capaz de revelar, por ejemplo, que el problema del infinito no se sustentaba en ningún significado intuitivo y que una investigación más detallada al respecto tampoco tenía sentido: "por todas partes solo hay cosas finitas" (Hilbert 2005c, p.1160). Por esta razón, tal como hemos señalado al principio de este trabajo, la perspectiva epistemológica de Hilbert se caracteriza por afirmar un "razonamiento finitista".

Respecto de las ciencias naturales, según Hilbert, también debemos asumir con estas teorías que únicamente hay cosas finitas: no hay velocidad infinita ni ninguna fuerza o efecto que se propague infinitamente rápido por el espacio. Todavía más, tal efecto en sí mismo es de una naturaleza discreta, solo existen quanta (cantidades). No hay absolutamente nada continuo que se pueda dividir infinitamente. Incluso la luz tiene la estructura atómica, al igual que los quanta de acción. Hilbert creía firme- 
mente en que incluso el espacio tenía una extensión finita: "un día los astrónomos serán capaces de decirnos cuántos kilómetros tiene de largo, alto y ancho" (Hilbert 2005c, p.1159). Hablamos de infinitud o de lo infinito porque es la negación de una condición que prevalece en todas partes, es una abstracción gigantesca practicable solo a través de la aplicación consciente o inconsciente del método axiomático.

Sin embargo, Hilbert sostendrá que, a pesar de la evidencia de la importancia del conocimiento abstracto y de las leyes de la lógica para la fundamentación de todo nuestro conocimiento, no todo el conocimiento proviene de la experiencia y de la deducción: tenemos también una fuente a priori de la realidad (Hilbert 2005c, pp.161-2). En este sentido volverá a rescatar a la intuición, pero no esa intuición sin base de la que depende la construcción geométrica de los conceptos o nociones primitivas de Euclides, sino de una especie de intuición o visión intuitiva [Anschaulicher Einsicht] en la que descansa el conocimiento abstracto de las matemáticas. Este es un "cierto punto de vista a priori intuitivo" que necesitamos para la construcción de nuestras teorías, y sobre todo de la teoría de los números. Con ello, Hilbert propone rescatar la idea más general y fundamental de la epistemología kantiana: el problema filosófico de la determinación intuitiva, o, en otras palabras, "la condición de posibilidad del conocimiento conceptual y de toda experiencia". No deja de llamar la atención la admiración que Hilbert tiene por Kant en estos pasajes, coterráneo suyo. Según Hilbert, la investigación de tal problema es lo que ha venido haciendo en cada uno de sus trabajos: "[c]reo que en esencia esto ha ocurrido en mis investigaciones sobre los principios de las matemáticas" (Hilbert 2005c, p.1162). Para Hilbert, el a priori es una perspectiva fundamental, es la expresión de ciertas condiciones previas indispensables de pensamiento y experiencia. Toda fundamentación del conocimiento - sobre todo el de las matemáticas - debe partir de la obtención y especificación de las proposiciones que provienen de esa fuente a priori. El sistema de conocimiento que tengamos debe suponer y esclarecer dichas proposiciones.

No obstante, Hilbert (2005c) señala que Kant sobrestimó el papel y el alcance de esa fuente a priori. Sostenía que era necesario establecer los límites para que lo $a$ priori no sobrepase aquello que únicamente necesita de la fuente de la experiencia como fundamento de conocimiento. Según Hilbert, Kant estaba familiarizado con la teoría matemática y física del espacio de su época, lo que se demostraba en sus ideas sobre - y sus ejemplos de - las representaciones de número, secuencia y cantidad, específicamente en el tratamiento de la intuición inmediata que podíamos tener de esas representaciones. Kant pensaba que las representaciones (Vorstellungen) que uno podía tener del espacio y el tiempo eran de una inmediata y general aplicación a la realidad. En su época, existía la concepción de que la teoría del espacio y del tiempo (la geometría), al igual que la aritmética, precedía a todo el conocimiento de la naturaleza. Pero incluso antes de que el desarrollo de la física obligara a lo contrario, este punto de vista kantiano ya había sido abandonado por Riemann y Helmholtz 
(Corry 2004a, pp.25-54). Según Hilbert, con mucha razón porque para la geometría no hay otra cosa más que no sea parte de la estructura total de los conceptos físicos, que modela las posibles relaciones de posición entre los cuerpos rígidos en el mundo de las cosas reales. Que haya cuerpos rígidos móviles y cuáles son sus relaciones posicionales es únicamente una cuestión de experiencia. Hilbert (2005c, pp.1162-3) decía compartir la misma opinión que Gauss de que la proposición 'la suma de los ángulos de un triángulo es igual a dos ángulos rectos' y, así también, que la validez del axioma de las paralelas estaban a punto de ser determinados, o refutados, por el avance de la experimentación científica.

Si, por ejemplo, todos los datos expresados por los teoremas sobre congruencia estuvieran de acuerdo con la experiencia, pero si, por el contrario, la suma de los ángulos de un triángulo construido a partir de barras rígidas llegara a ser menos de dos ángulos rectos, nadie golpearía con la idea de que el axioma de las paralelas es válido en el espacio de los cuerpos reales (Hilbert 2005c, p.1162).

Para Hilbert se hará necesario estudiar qué es lo que puede incluirse en el dominio de lo a priori, ya que algunos contenidos que antes se contaban dentro de ese dominio ahora son tenidos por incorrectos (sobre todo en geometría). El ejemplo más esclarecedor al respecto, según Hilbert, es la idea del presente absoluto. Tendemos a creer que existe un presente absoluto, pero no existe; no es más que nuestra sensación de las distancias cortas y los movimientos lentos. Pero aun grandes pensadores como Newton y Kant trataban de sostenerlo. Newton afirmaba que el tiempo absoluto verdadero, en virtud de su naturaleza, fluye por sí mismo de manera uniforme y sin relación con cualquier objeto. Para Hilbert, Kant no fue crítico en este punto, aceptó simplemente esta concepción de Newton. Solo Einstein nos liberaría definitivamente de ese prejuicio. La teoría del a priori, que fue llevada demasiado lejos por Kant, quedó reducida al absurdo por el avance de la física de Einstein. Por ejemplo, Hilbert creía que, para la concepción de un tiempo absoluto, la teoría de Einstein demostraba de manera sencilla que el teorema de la suma de velocidades era incorrecto (Corry 2004a, Capítulo 5, pp.22-286).

En efecto, lo más importante para Hilbert es que con Einstein, inevitablemente, se debe asumir que la geometría no es más que una rama de la física. Por ello, las verdades geométricas no son en modo alguno diferentes a las verdades físicas (Hilbert 2005c, p.1163): el teorema de Pitágoras y las leyes de atracción de Newton por ejemplo - estarán esencialmente relacionadas, ya que ambas están gobernadas por un mismo concepto fundamental, que en este caso es el concepto de "potencia". Entonces, ¿qué clase de dominio de conceptos (a priori) debe ser este? Más aún, con la teoría de gravitación de Einstein ambas leyes no solo tendrán el mismo carácter $a$ priori - gracias a que permanecen bajo un mismo concepto — sino que serán ahora 
partes de una y la misma ley general. Por este motivo se hará necesario especificar el dominio de lo a priori. Según Hilbert, el único camino para la matemática.

Hilbert sostenía que había quedado demostrado, desde hace bastante tiempo, que tenemos un acceso efectivo a ese dominio a priori (que determina la realidad) por medio del instrumento de las matemáticas. Ellas construyen un puente de conexión entre la teoría y la práctica, entre el pensamiento y la observación (Hilbert 2005c, p.1163). Ya Galileo decía que solo el hombre puede entender la naturaleza con la que se ha familiarizado a través de su lengua y con los signos en los que ella nos habla: la matemática. "Kant hizo la siguiente observación: "sostengo que en toda ciencia natural en particular, la cantidad de matemáticas que contiene es equivalente a la cantidad de ciencia genuina que podemos hallar en ella"' (Hilbert 2005c, p.1164).

En esta concepción epistemológica, la única manera de concebir la teoría de la naturaleza es como un compendio del núcleo matemático que se extrae de la naturaleza misma. Para Hilbert, sin matemáticas no hay física ni astronomía modernas. Pero el examen que realiza en Lógica y el conocimiento de la naturaleza (1930) muestra que los matemáticos aún no habían comprendido bien este asunto - sobre todo era muy crítico con Gauss (aunque salva a Poincaré y Jacobi) —, no quieren permitir el avance de las matemáticas como ciencia fundamental para el conocimiento de la naturaleza (2005c, p.1165). Su principal error es haber introducido objetos primitivos en los sistemas matemáticos a partir de definiciones (principalmente en geometría) sin fundamentos, sin prueba de su existencia, basados en intuiciones matemáticas que imposibilitan la fundamentación por el riesgo que implican para la consistencia de estos. La única vía posible es a través de la axiomatización de los sistemas, basada en ese "cierto punto de vista a priori intuitivo" que necesitamos para la construcción de nuestras teorías. La lógica aparece entonces para determinar el conjunto de proposiciones verdaderas de las cuales se debe comenzar la construcción del conocimiento, partiendo por el conocimiento matemático. La matemática se servirá de la lógica ya que el método axiomático pertenece a la lógica (Hilbert 2005c, p.159).

En la Fundamentación de la teoría elemental de los números, una obra más tardía de 1931, que ha resultado excesivamente filosófica para la tradición matemática (Corry 2004a, pp.287-316), Hilbert trata de aclarar la naturaleza general del conocimiento. Señalará que el a priori al que apela en su teoría de la prueba no es ni más ni menos que el fundamento básico de lo que él concibe como lo finitista: un modo fundamental del pensamiento [Grundeinstellung] o modo finito del pensamiento. Este modo es algo que ya nos ha sido dado por adelantado a nuestra facultad de representación. Lo que se nos ha dado a priori son "objetos extra-lógicos concretos que existen intuitivamente como una experiencia inmediata antes de todo pensamiento" (Hilbert 2005b, p.1150).

Pero Hilbert tiene una exigencia: los objetos extra-lógicos, que existen a priori para ser dados al pensamiento como evidencias inmediatas - como los puntos y líneas 
de la geometría -, deben ser completamente o "lógicamente" analizables en todas sus partes y relaciones. Todo lo que se diga o deduzca a partir de ellos debe poder probarse. Es la única forma de asegurar la intuición. Un sistema que involucre tales objetos debe garantizar entonces que sus proposiciones de base, de las cuales estos objetos extra-lógicos son sus contenidos, no puedan reducirse a otras, esto quiere decir, que esté garantizada su independencia y consistencia dentro del mismo conjunto de proposiciones base: los axiomas. El modo fundamental de captar dichos objetos es el a priori del pensamiento, que es necesario para las matemáticas y el conocimiento en general. Por este motivo, el a priori al que apela Hilbert es distinto del a priori kantiano, aunque le deba mucho: los conocimientos a priori son aquellas intuiciones, así como las ideas de la lógica, que consiguen el marco del modo de pensamiento finito, la axiomática (Hilbert 2005b, pp.1150-1). Lo que se realiza dentro de ese marco sobre los objetos extra-lógicos es una operación de separación o abstracción: análisis. Según esto, la diferencia con Kant es clara: una operación de este tipo prescinde de las categorías de espacio y tiempo. En el caso de la teoría de los números, el carácter intuitivo que Kant atribuye en la Crítica de la razón pura (1997, pp.46-56) a esos objetos se funda en las intuiciones del espacio y del tiempo, que para Hilbert son objeto de la experiencia sensible. Por este motivo, la única vía de fundamentación de nuestro conocimiento científico será a través del método axiomático, el cual emparenta el análisis lógico con el instrumento y la fuente del conocimiento, que son las matemáticas.

\section{Conclusión}

Según lo expuesto, la teoría de la prueba de Hilbert es una teoría donde las proposiciones básicas del sistema se exponen al inicio de su formulación. Esas proposiciones contienen los conceptos y nociones que especifican los objetos con los cuales trata la teoría. A partir de dichas proposiciones se deriva lógicamente el resto del contenido a través de las pruebas, y son estas mismas pruebas las que justifican la existencia de todos los objetos de la teoría. Si aplicamos la teoría de la prueba al resto de las ciencias, todo el conocimiento queda reducido a unas pocas proposiciones de las que se deriva lógicamente. La intuición (por ejemplo, en el caso de la geometría) o la experiencia (por ejemplo, en el caso de la física) que tenemos de esas proposiciones y sus consecuencias quedan determinadas por la validez y la consistencia del sistema que describe y explica ese ámbito de la realidad. El conjunto de proposiciones fundantes - los axiomas - es finito, al igual que todas las proposiciones que se derivan de este. El sistema debe entenderse como un sistema cerrado, válido, coherente y consistente. Y el método axiomático no hará más que probar la no-contradicción dentro del sistema, que es un principio manejable solo desde un universo matemático finito. 
La configuración del sistema que intenta fundar Hilbert necesita de un dominio de proposiciones verdaderas a las cuales recurre el conocimiento matemático. De ellas parte el conocimiento matemático. Recuérdese que Hilbert señala que las matemáticas deberán entenderse ahora como un "depósito de fórmulas" donde conviven las fórmulas con signos lógicos y las fórmulas matemáticas ordinarias, o solo con símbolos matemáticos. Los axiomas son fórmulas (o proposiciones) que son como los ladrillos (o piedras) fundantes de un edificio, que son captados gracias a la lógica que entrega su método axiomático a las matemáticas. En esta perspectiva de la lógica afirmará entonces que la fuente del conocimiento se haya en ese apriorismo de proposiciones. Como enfoque epistemológico debe sostener que, como último término, la "unidad mínima del conocimiento" es la proposición lógica, que - según Hilbert - obtenemos gracias a la intuición. Esta hipótesis reviste de sentido a la pregunta por el papel que juega la lógica para el conocimiento científico, especialmente para su reconstrucción formal.

El programa de Hilbert, como es sabido, fracasó como aclaración completa de los problemas fundamentales de las matemáticas a través de las pruebas finitistas de consistencia. Algunos autores, como Prawitz (1965) y Corry (2004b) creen que el principal error de Hilbert fue no tratar con las pruebas matemáticas en sí mismas. En efecto, Hilbert se centró solo en las pruebas que contenían las proposiciones con símbolos lógicos que él pensaba que fundamentaban los sistemas. Esto quiere decir, que solo le preocupó los problemas de la fundamentación de las matemáticas y no el desarrollo de su práctica, especialmente en los ejemplos de la geometría y de la teoría fundamental de los números. Su intención principal podría entenderse como la elaboración de una teoría general de los métodos de prueba para las matemáticas (Grattan-Guinness 2000, pp.206-18).

\section{Referencias}

Blanchette, P. 2012. The Frege-Hilbert Controversy. In: E. N. Zalta (ed.) Stanford Encyclopedia of Philosophy. https://plato.stanford.edu/entries/frege-hilbert/. Access: 17/08/2018.

Bolzano, B. 1973[1837]. Theory of Science. Dordrecht: D. Reidel Publishing Company.

Buss, S. R. 1998. An Introduction to Proof Theory. In: Samuel R. Buss (ed.) Handbook of Proof Theory. Vol. 137, pp.1-78. Studies in Logic and The Foundations of Mathematics Book Series. Oxford: Elsevier.

Cantor, G. 1955. Contributions to the Founding of the Theory of Transfinite Numbers. New York: Dover Publications.

Cassini, A. 2013. El juego de los principios. Una introducción al método axiomático. Buenos Aires: AZ Editora.

Cohen, D. W. 1989. An Introduction to Hilbert Space and Quantum Logic. New York: SpringerVerlag. 
Corry, L. 2004a. David Hilbert and the Axiomatization of Physics (1989-1918). From Grundlagen der Geometrie to Grundlagen der Physik. Dordrecht: Springer- Science+Business Media, B.V.

- 2004b. Modern Algebra and the Rise of Mathematical Structures. Berlin: Sgringer Basel AG.

da Costa, N. C. A.; French, S. 2003. Science and Partial Truth: A Unitary Approach to Models and Scientific Reasoning. Oxford: Oxford University Press.

Detlefsen, M. 1986. Hilbert's Program: An Essay on Mathematical Instrumentalism. Synthese Library - Studies in Epistemology, Logic, Methodology and Philosophy of Science, Volume 182. Dordrecht: Springer Science+Business Media.

Díez, J. A.; Moulines, C. U. 2008. Fundamentos de filosofía de la ciencia. Barcelona: Editorial Ariel.

Euclides 1991. Elementos. Libros I-IV. Madrid: Editorial Gredos.

Ewald, W. B. 1996. From Kant to Hilbert: A Source Book in the Foundations of Mathematics, Vol. II. Oxford: Clarendon Press.

Feferman, S. 2008. Axioms for Determinateness and Truth. Review of Symbolic Logic 1: 20417.

Frege, G. 1972[1879]. Conceptografía. Ciudad de México: Editorial Universidad Nacional Autónoma de México.

Gabriel, G.; Hermes, H.; Kambartel, F.; Thiel, C.; Veraart, A.; McGuinness, B.; Kaal, H. (eds.) 1980. Gottlob Frege. Philosophical and Mathematical Correspondence. Oxford: Basil Blackwell.

Gödel, K. 2006. Sobre sentencias formalmente indecidibles de Principia Mathematica y sistemas afines. In: J. Mosterín (ed.) Kurt Gödel. Obras completas, pp.53-89. Madrid: Alianza Editorial.

Grattan-Guinness, I. 2000. The Search for Mathematical Roots, 1870-1940: Logics, Set Theories and the Foundations of Mathematics from Cantor through Russell to Gödel. Oxford: Princeton University Press.

Haack, S. 2009. Philosophy of Logics. Cambridge: Cambridge University Press.

Halbach, V. 2011. Axiomatic Theories of Truth. Cambridge: Cambridge University Press.

Heyting, A. 1971. Intuitionism. An Introduction, Studies in Logic and the Foundations of Mathematics. Amsterdam: North-Holland Publishing Company.

Hilbert, D. 1905. On the Foundations of Logic and Arithmetic. The Monist 15(3): 338-52.

—. 1950[1899]. The Foundations of Geometry. Illinois: The Open Court Publishing Company.

—.1993[1899-1930]. Fundamentos de las matemáticas. México D.F.: Mathema. 2000[1900-1901]. Mathematical Problems. Bulletin-American Mathematical Society 37(4): 407-36.

- 2005a[1918]. Axiomatic Thought. In: W. Ewald (ed.) From Kant to Hilbert: A Source Book in the Foundations of Mathematics. Volume II, pp.1105-15. Oxford: Clarendon Press. - 2005b[1931]. The Grounding of Elementary Number Theory. In: W. Ewald (ed.) From Kant to Hilbert: A Source Book in the Foundations of Mathematics. Volume II, pp.1157-65. Oxford: Clarendon Press.

- 2005c[1930]. Logic and the Knowledge of Nature. In: W. Ewald (ed.) From Kant to Hilbert: A Source Book in the Foundations of Mathematics. Vol. II, pp.1157-65. Oxford: 
Clarendon Press.

- 2005d[1923]. The Logical Foundations of Mathematics. In: W. Ewald (ed.) From Kant to Hilbert: A Source Book in the Foundations of Mathematics. Volume II, pp.1134-48. Oxford: Clarendon Press.

Hilbert, D.; Ackermann, W. 1950. Principles of Mathematical Logic. New York: Chelsea Publishing Company.

Hintikka, J. 1988. On the Development of the Model-Theoretic Viewpoint in Logical Theory. Synthese 77(1): 1-36.

Huertas, A.; Manzano, M. 2017. Teoría de conjuntos. https://webs.ucm.es/info/pslogica/teoriaconjuntos.pdf. Access: 14/10/2018.

Kant, I. 1997. Crítica de la razón pura. Madrid: Alfaguara.

Kitcher, P. 1976. Hilbert's Epistemology. Philosophy of Science 43(1): 99-115.

Kronecker, L. 1996[1887]. On the Concept of Number. In: W. B. Ewald (ed.) From Kant to Hilbert: A Source Book in the Foundations of Mathematics. Volume II, pp.947-55. Oxford: Clarendon Press.

Mancosu, P. 2010. The Adventure of Reason: Interplay Between Philosophy of Mathematics and Mathematical Logic, 1900-1940. Oxford: Oxford University Press.

Mosterín, J. 1980. La polémica entre Frege y Hilbert acerca del método axiomático. Teorema 10(4): 287-306.

Mueller, I. 1981. Philosophy of Mathematics and Deductive Structure in Euclid's Elements. Massachusetts: The MIT Press.

Prawitz, D. 1965. Natural Deduction, a Proof-Theoretical Study. Stockholm: Almqvist \& Wiksell.

Resnik, M. D. 1974. On the Philosophical Significance of Consistency Proofs. Journal of Philosophical Logic 3(1-2): 133-47.

Rowe, D. 2000. The Calm Before the Storm: Hilbert's Early Views on Foundations. In: F. E. A. Hendricks (ed.) Proof Theory. History and Philosophical Significance, pp.55-94. London: Kluwer Academic Publisher.

Sieg, W. 2013. Hilbert's Programs and Beyond. Oxford: Oxford University Press.

van Benthem, J. 2001. Games in Dynamic-Epistemic Logic. Bulletin of Economic Research 53(4): 219-48.

- 2014. Logic in Games. Massachusetts: MIT Press.

Zach, R. 2015. Hilbert's Program. In: E. N. Zalta (ed.) Stanford Encyclopedia of Philosophy. https://plato.stanford.edu/entries/hilbert-program/. Access: 16/11/2018.

\section{Notas}

${ }^{1}$ Habiéndose superado la arremetida de los enfoques historicistas en filosofía de la ciencia - donde la lógica y la axiomatización eran vistas como "un mero despliegue técnico aplicado sin mucho sentido" - , los enfoques semánticos como la metateoría estructuralista o el realismo estructural, y sus consecuentes despliegues actuales, siguen defendiendo que la axiomatización, aun cuando se reconozca como defectuosa, "es un componente fundamental para la filosofía de la ciencia, por su papel en la introducción de claridad con respecto a los 
conceptos básicos de una teoría, por su ayuda en la comparación teórica (anatema, por supuesto, para aquellos que cree en la inconmensurabilidad), por la forma en que puede abrir una teoría a técnicas matemáticas potencialmente fructíferas, y por su utilidad para resolver ciertas disputas filosóficas" (Da Costa 2003, p.23; cf. Díez y Moulines 2008, capítulos 1 y 10). Los recursos y métodos formales siguen siendo utilizados para el análisis de teorías científicas, obviamente con una riqueza mayor que la lógica de primer orden favorita de la perspectiva clásica (concepción heredada), esto es, utilizando teoría de modelos, teoría de conjuntos, lógica paraconsistente, topología, entre otras.

${ }^{2}$ Cfr. 3§: "Normal Form for Logical Expressions".

${ }^{3}$ Teorema 1: cualquier teoría aritmética recursiva que sea consistente es incompleta; Teorema 2: en toda teoría aritmética recursiva consistente $T$, la fórmula consistente $T$ no es un teorema (Gödel 2006, pp.53-89).

${ }^{4}$ Para una lista más acabada de ejemplos cfr. Grattan-Guinness (2000).

${ }^{5}$ En los manuscritos originales de dicha conferencia, pronunciada en el Segundo Congreso Internacional de Matemática realizado en París en agosto de 1900, Hilbert enumera un total de 23 problemas a resolver y con los cuales debe ponerse a prueba el método axiomático que anticipa. Su problema número seis se refiere a la fundamentación de las ciencias naturales, dice aquí: "[t]he investigations on the foundations of geometry suggest the problem: To treat in the same manner, by means of axioms, those physical sciences in which mathematics plays an important part; in the first rank are the theory of probabilities and mechanics". (Hilbert 2000, p.418). Esta idea tuvo un impacto significativo para el Círculo de Viena y para toda la perspectiva clásica de la filosofía de la ciencia que hoy denominamos "concepción heredada". A pesar de ello, la complejidad y confusiones del pensamiento filosófico Hilbert — como veremos a continuación - advierten del problema que reviste caracterizarlo dentro del proyecto filosófico clásico del empirismo lógico y sus variantes. Pero claramente, tal como ocurre con el primer Wittgenstein, su influencia es indiscutida.

6 "In the meantime, while the creative power of pure reason is at work, the outer world again comes into play, forces upon us new questions from actual experience, opens up new branches of mathematics, and while we seek to conquer these new fields of knowledge for the realm of pure thought, we often find the answers to old unsolved problems and thus at the same time advance most successfully the old theories. And it seems to me that the numerous and surprising analogies and that apparently prearranged harmony which the mathematician so often perceives in the questions, methods and ideas of the various branches of his science, have their origin in this ever-recurring interplay between thought and experience". (Hilbert 2000, p.409)

${ }^{7}$ L. Kronecker (1823-1891) sostuvo con mucha fuerza el finitismo en matemática, cuya idea principal es que un objeto matemático no existe a menos que sea construido partiendo de números naturales en un número finito de pasos (por ello se prescinde de los irracionales e imaginarios), por lo que se le considera precursor del intuicionismo (Kronecker 1996).

${ }^{8}$ Fundamentos de la geometría (1899) y Fundamentos de las matemáticas (1927).

${ }^{9} \mathrm{En}$ su introducción escribe: "The following investigation is a new attempt to choose for geometry a simple and complete set of independent axioms and to deduce from these the most important geometrical theorems in such a manner as to bring out as clearly as possible the significance of the different groups of axioms and the scope of the conclusions to be derived from the individual axioms" (Hilbert y Ackermann 1950, p.1). 
${ }^{10}$ Fue la primera máquina lógica que podía calcular con el álgebra de Boole para resolver algunos problemas lógicos. Fue creada por el científico William Stanley Jevons en 1869. Se le llama así porque tenía la forma de un pequeño piano de pared.

11 "If contradictory attributes be assigned to a concept, I say, that mathematically the concept does not exist. So, for example, a real number whose square is 1 does not exist mathematically. But if it can be proved that the attributes assigned to the concept can never lead to a contradiction by the application of a finite number of logical processes, I say that the mathematical existence of the concept (for example, of a number or a function which satisfies certain conditions) is thereby proved". (Hilbert 2000, p.414).

${ }^{12} \mathrm{El}$ trabajo más representativo de Bolzano al respecto es "Purely analytic proof of the theorem that between any two values which give results of opposite sign there lies at least one real root of the equation", de 1817. El trabajo de Boole es "The mathematical analysis of logic, being an essay towards a calculus of deductive reasoning", de 1847. Y los trabajos más relevantes de Peirce al respecto son "On the logic of number", de 1881, y "On the algebra of logic: a contribution to the philosophy of notation", de 1885. Estos trabajos han sido recopilados en Ewald (1996).

13“Contribuciones a la fundamentación de la teoría de conjuntos transfinitos".

\section{Agradecimientos}

Agradezco a la profesora María José Frápolli (Universidad de Granada, España) por sus comentarios, sugerencias y apoyo incondicional durante la elaboración de este trabajo. También agradezco a Diego Fernandes (Universidade Federal de Goiás, Brasil) y a Daniel Álvarez Domínguez (Universidad de Salamanca, España) por sus sugerencias y oportunas correcciones. Este trabajo ha sido financiado por CONICYT PFCHA/DOCTORADO BECAS CHILE/2015 72160368 . 